\title{
Using Geographic Information Systems (GIS) for UAV Landings and UGV Navigation
}

\author{
Nathan Rackliffe, Holly A. Yanco, and Jennifer Casper \\ The MITRE Corporation, Bedford, Massachusetts 01730-1420 \\ nrackliffe@mitre.org, hyanco@mitre.org, jcasper@mitre.org
}

\begin{abstract}
Unmanned air and ground platforms are not currently designed to process contextual information (e.g., land use, building types and time of day) to assist in landing and navigation. Instead, a human operator often provides manuallygathered and synthesized contextual input through control commands. This paper describes research that explores the integration of geographic information system (GIS) data with sensor data to enable (1) unmanned aerial vehicles (UAV) to locate safe emergency landing locations without operator intervention, and (2) unmanned ground vehicles (UGV) to incorporate contextual GIS information for navigation. Informal evaluation of the system revealed the potential to impact navigation and emergency landings through the additional contextual information provided.
\end{abstract}

\section{INTRODUCTION}

The use of contextual information such as the location and uses of buildings in the area can improve the autonomous capabilities of unmanned systems. However, at this time, unmanned air and ground vehicles are not currently designed to process contextual information to aid in landing and navigation. For unmanned aerial vehicles (UAV) operating in the national airspace (NAS), it is critical to be able to find safe emergency landing zones that avoid people, houses, and populated areas, particularly in cases where the UAV has lost its link with the operator on the ground. For unmanned ground vehicles (UGV), sensors and processing provide information on physical objects, terrain and mapping, but not contextual information about the area (i.e., whether a building is a school, hospital, shopping mall, etc.); this contextual information can be used to improve the performance of a UGV and increase its autonomous capabilities.

Autonomous systems used by the military, including UAVs and UGVs, require frequent monitoring and intervention from human operators. Operators take into account any contextual information they can gather. In addition, missions are planned for these systems at varying levels of granularity. Some systems are given an end goal, some are given waypoints, and others require additional detail which might include teleoperation. Situations may arise during the execution of these plans that require dynamic replanning. An example of such an event for a UAV would be a failure requiring an emergency landing; an example of such an event for a UGV would be encountering a hazard. Contextual information such as the time of day and type of environment could inform

Approved for Public Release: 11-0082. Distribution Unlimited. navigation and emergency landing plans. Additionally, the use of GIS data together with sensor data gathered by autonomous systems could potentially improve the systems' reaction to anomalous situations that arise and improve navigation routes.

The objective of this effort is to explore the integration of GIS data with sensor data and time of day information to enable (1) UAVs to locate safe emergency landing locations without operator intervention, and (2) UGVs to incorporate contextual GIS information for navigation. Ultimately, the combination of GIS maps that include precomputed probabilities for preferred landing sites or navigation routes with current sensor data will help inform and improve the operation of UGVs and UAVs.

Preliminary evaluation of our system, which integrates GIS and sensor data, reveals potential impact through more efficient navigation and the ability to locate safe landing zones. This effort builds the foundation for employing GIS data streams in UAV landing and UGV navigation. The platform agnostic nature of our effort, which focuses on the fusion and processing of multiple layers of GIS data according to a specified mission profile, could be used for any type of autonomous system. This effort not only impacts the UAV and UGV fields, but can also apply to other fields such as data analysis interfaces.

This paper presents an approach to incorporating GIS that revolves around building probability and cost surface maps. The architecture includes Environmental Systems Research Institute (ESRI) ArcGIS components for GIS data processing. ${ }^{1}$ Our system was designed to take in the GIS data, process it according to mission parameters utilizing ArcGIS, and output probability maps which can either indicate where to land or navigate. ArcGIS Server supports various output formats. In this paper, the maps are presented as heat-map style images. An overview of the architecture is shown in Figure 1.

We have taken a platform-agnostic approach as the maps produced may be used for UGVs, UAVs and other types of existing systems. Three phases are needed to create the maps: (1) data collection, (2) data conversion, and (3) geo-processing. In our current system, steps one and two are manual; the architecture executes step three.

\section{RELATED WORK}

A wide variety of GIS data, covering many different types of features, is publicly available. The level of detail, age

${ }^{1}$ ESRI ArcGIS Information: http://www.esri.com/software/arcgis/index.
html 


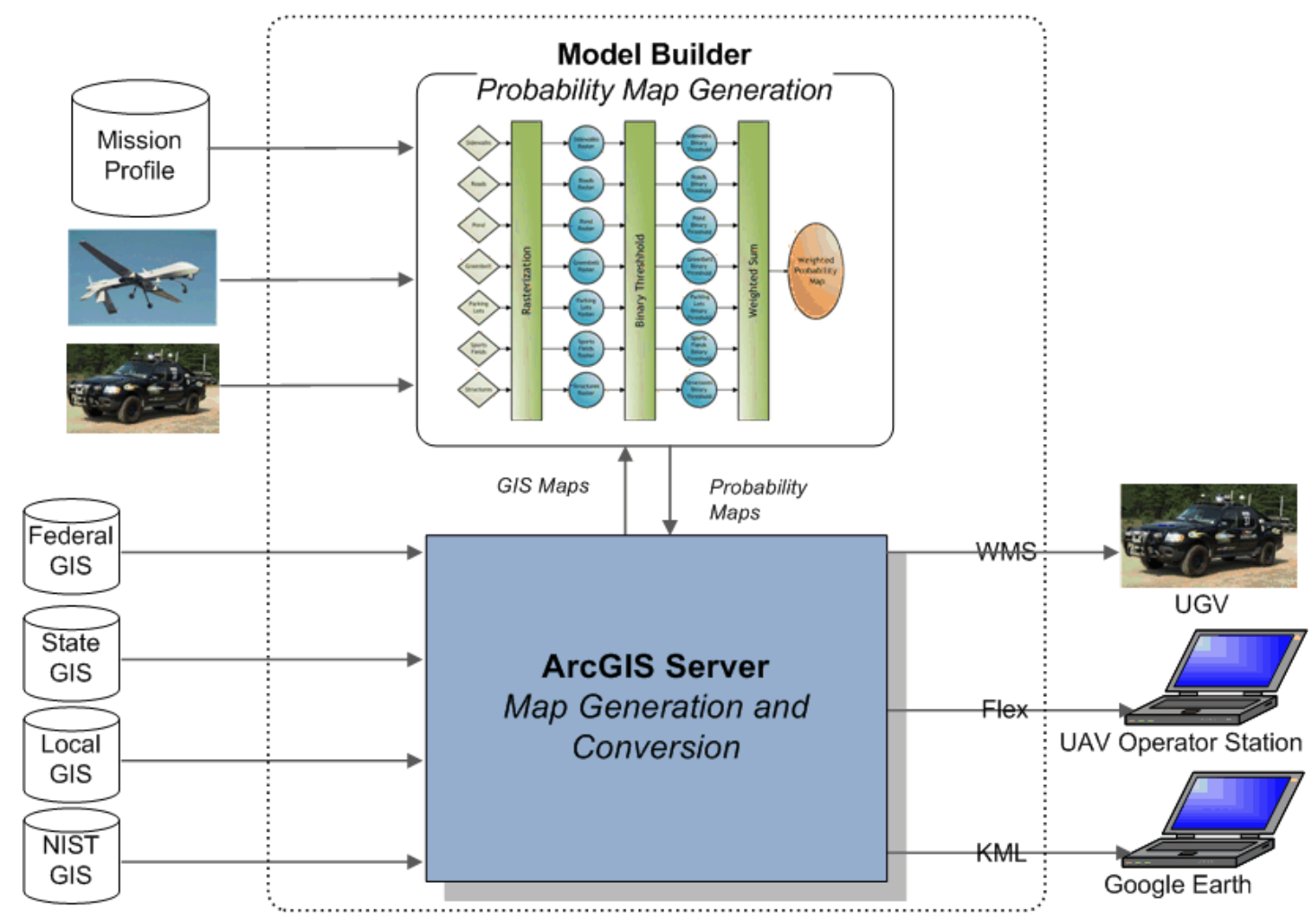

Fig. 1. Overview of architecture incorporating components of ESRI ArcGIS.

of the data, and accuracy varies for each data set. Despite the potential imperfections in the GIS data, the information provides an additional level of detail to inform autonomous systems. Although GIS data is typically two dimensional (2D), three dimensional (3D) GIS data is being produced through highly accurate 3D environment models. The authors in [1] explored the use of 3D GIS data for robot localization. The work described in the following paragraphs, as well as our research, makes use of 2D GIS data.

The use of GIS data within the robotics field has been explored over the last decade and grown in recent years as GISs have evolved. ${ }^{2}$ One of the earliest uses of GIS for robots was in the design of a mechanical guide machine for the visually impaired [2]. Although the machine did not have the traversing capabilities of a dog, it offered additional navigation through dynamic map creation that incorporated GIS for contextual information. The authors in [3] highlight the importance of contextual information for mobile robot navigation. Satellite and mapping imagery provided the means to repair spatial inaccuracies for waypoint navigation.

A common use of GIS data is for robot localization in outdoor environments. Advanced sensors enable perception of the immediate surroundings, although context is unknown. Correlating features or landmarks extracted from sensors with GIS data allows aligning. GIS is useful in verifying robot position in the world, in conjunction with GPS in some

\footnotetext{
${ }^{2}$ An annotated bibliography may be viewed here: http://dusk.geo.orst.edu/ gis/student_bibs/nehmer/
}

cases. The use of GIS for landmark localization to aid vehicle navigation has been used by many researchers (e.g., [4]-[10]). In [7], an approach for tracking a specific feature (roads) for autonomous vehicle navigation is described. A road model is used to determine if sensor data is picking up road edges; GIS and GPS data are employed for verification and localization.

GISs provide an inherent means to share geospatial data. The research in [11] takes an alternative approach to incorporating GIS. Instead of consuming the GIS data, GIS was used as a means of sharing information on the status of a disaster environment. The data retrieved from the robots employed to explore the disaster area was transmitted to a GIS via a designated data format over wireless communications link. This information may then be consumed by other systems or robots. This approach results in a view of the environment that is dynamically updated.

GIS data can expand to large areas and encompass an entire space in which a team of robots are operating. The GIS data covering the operating area provides a shared view of the environment. The authors in [9], [12] highlight the use of GIS data for cooperative map building and navigation. Localization is coordinating through use of the GIS maps. Each robot updates their position and findings to the common space for sharing with the other systems.

A benefit of incorporating GIS with robotics is that the systems support human readable formats: robotics ultimately involves human interaction at some level and GIS data supports human-robot interactions; a point stressed in [9]. 
Our effort is focused on exploring the use of GIS with sensor data for constructing probability maps for UGV navigation and UAV landings. The authors in [13] presented a method to build a probability map using local sensors and known environment statistics intended for UGVs and UAVs. Our effort expands on this concept through the addition of GIS data. This contextual information supports more detailed probability map construction.

\section{Architecture Design}

A system that leverages GIS data, including ours, involves several processing steps. First, data needs to be collected from available sources, including federal, state and local agencies. Next, the data needs to be converted from the source formats into common projections and extents so it can be compared. Finally, the data is geoprocessed into the usable products. In our work, the products are either probability maps or cost surfaces to be used by unmanned autonomous systems to make navigation decisions. The following subsections walk through the three steps in processing GIS data for use by unmanned systems.

\section{A. Data Collection}

A wide variety of GIS data is publicly available, covering many different types of features. The level of detail for each data set varies as well as the age of the data and its accuracy. We can take advantage of this meta-data to inform our probability modeling process; for example, more current data can be weighted more heavily than old data. GIS data can be roughly categorized into the following levels: global, federal, state, county-city, and organization; the advantages and disadvantages of each type are described below. For this effort, we used data from Texas A\&M University (TAMU). Their data is specific to their campus, but is of high enough granularity to be useful for unmanned vehicle navigation. ${ }^{3}$

1) Global Data: Data at the global level is usually very coarse grained and is generally only able to resolve features larger than 1 square kilometer. This granularity limits the utility of these layers for UGV operations, where the robot only rarely will cross from one tile to the next. It can be useful for UAV operations where a course-grained map can still provide some information. An advantage of these data sets is that you can achieve full global coverage with relatively small file sizes.

2) Federal Data: Data from federal agencies gives more detail about the features found within their boundaries. Many of the federal layers are released openly to the public and contain a wide variety of information such as terrain information and land use. While this data is finer grained than that in the global level, it is still mostly too coarse for UGV use, but can be useful for UAVs.

\footnotetext{
${ }^{3}$ The data was obtained from the following server: http://fcor.tamu.edu/ ?pageid $=77$.
}

3) State Data: Most states have a GIS office that coordinates the use of GIS data within their boundaries. Because they cover a much smaller area, the resolution of the data is usually superior to that obtained from the federal level. They also have data from state agencies that is tailored to the local area.

4) County-City Data: Generally, county-city data is the finest granularity level available. Most cities now use GIS systems to track tax parcels and for planning purposes. In some cases, they have the location of every permanent structure within their boundaries. While some of this data is freely available over the web, most GIS departments are not connected to the internet and will deliver the data on DVD for a reasonable fee.

5) Organization Data: For organizations of sufficient size (e.g., large corporations, universities, etc.), they usually will have their own GIS office that tracks their campus and its details. This data is almost always at a higher fidelity than available from any of the above sources. It generally contains at least the structure information and may include details as fine grained as individual tree and shrub placement.

\section{B. Data Conversion}

Agencies employ different data standards. It is important to convert the diverse data sources to a common format; ArcGIS provides tools to do this. This process generally needs to be done only once per data layer, as further updates to the layer will be able to leverage the same projection transformation. For UGVs, the area of operation is small enough that few problems arise. A UAV operating across multiple states would need to correlate many more data sources and require additional conversion before use.

For the examples shown in this paper, we used data covering the TAMU campus. The data from TAMU is in a North American Datum (NAD) 1983 Texas Central State Plane projection. The other layers used were projected to this common projection so that all layers could be compared directly. This process, while optional, speeds the geoprocessing step since you can then avoid the need to project each layer on-the-fly.

\section{Geoprocessing}

With the data in a common projection, it can be geoprocessed into the components that we want to include in the probability map. The first stage is to create a rasterized representation of each data layer. The ArcGIS tools allow this step to be scripted based on a set of parameters. Once the layers are rasterized, each layer of interest is thresholded into a binary raster with good and bad areas marked. The thresholding parameters are determined by the mission profile. For example, on the structures layer, which includes all of the buildings in the area, the buildings are marked as nontraversable, while everything else is marked as traversable. Future work could expand this classification beyond a simple binary threshold; our initial work was focused on the development of the architecture and a proof of concept. 


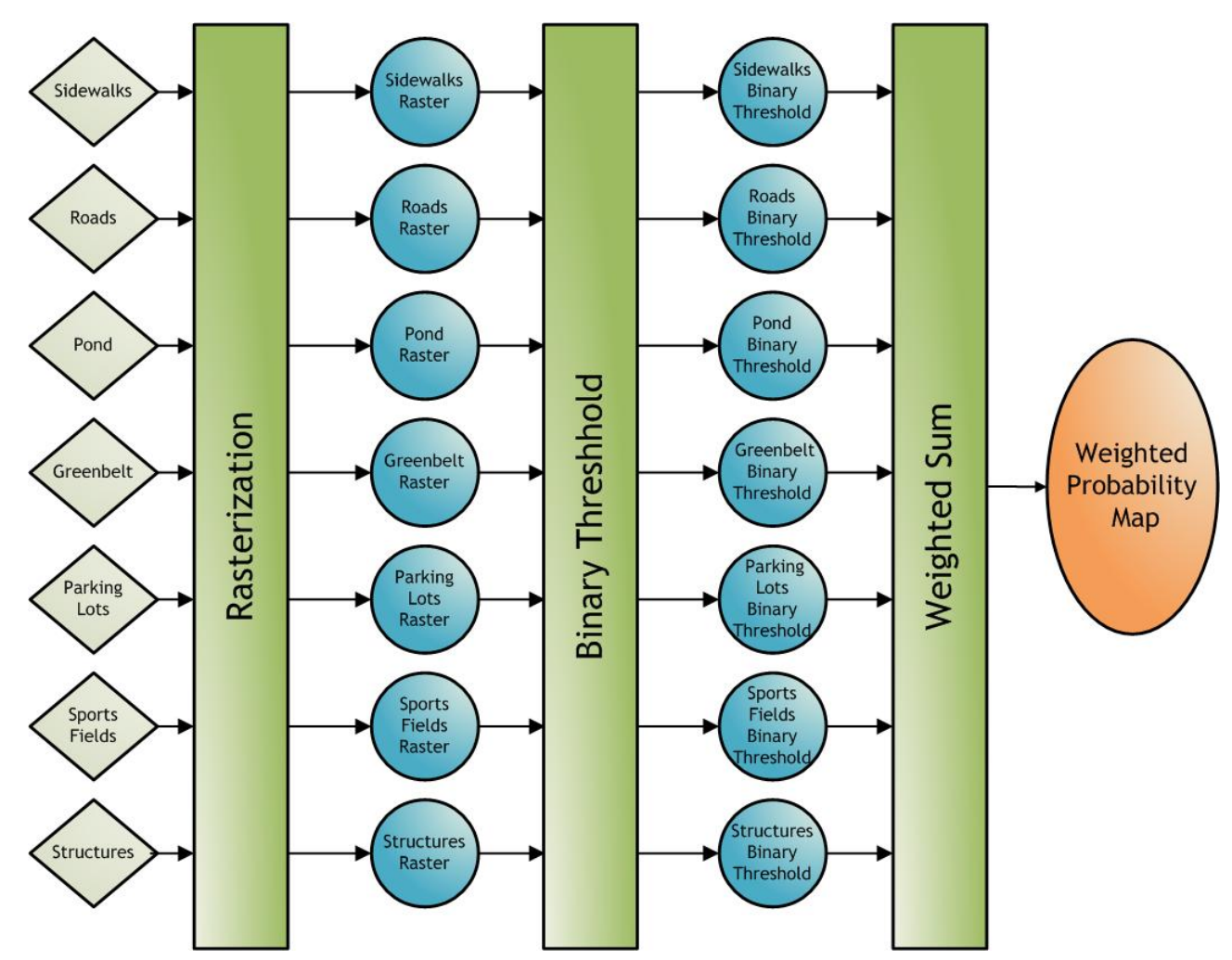

Fig. 2. Geoprocessing for this effort involves three phases: rasterization, thresholding, and computing a weighted sum. The result is a composite map representing probability of traversal or probability of minimized impact (depending upon a UGV or UAV application, respectively).

With all of the interesting layers rasterized and thresholded, the final geoprocessing steps are executed. A weighted sum is calculated from each of the component layers. The weights are selected according to the mission profile of the robot. For the UGV case, roads may be preferred for navigation, in which case the contribution of the traversable roadways will be increased. However, if the mission profile stated that the UGV should try to be stealthy by avoiding roads, the contribution of the roadways would be reduced. Buildings are generally to be avoided, for both UGVs and UAVs, and so are strongly weighted as non-traversable. However, for a patrol task for a $\mathrm{UGV}$, the area immediately around buildings could receive a higher weighting, resulting in the UGV system traveling near the buildings as it patrols.

The final composite layer is built up from these summed components and, if needed, clipped to the area of influence of the robot. This composite layer can be thought of as a probability of traversability (for UGVs) or probability of minimized impact (for UAVs) map for the area in question. The composite layer is added to the GIS server and is available to be served up in a wide variety of formats to clients. Figure 2 represents the geoprocessing workflow for this effort.

\section{Probability Models}

The probability models used for the geoprocessing step previously described are defined by the user through ArcGIS. The models may be adjusted on the fly as conditions change or additional information is gathered. Figure 3 represents the probability of minimized impact for an emergency UAV landing. The map on the left represents safe landing zones (green), unknown (yellow), and not-safe (red) for a small UAV during the weekday. The map on the right shows the safe, unknown, and not-safe zones for a small UAV during the weekend. Note the larger amounts of red due to likelihood of the area being used for sporting events on the weekends. The cone-shaped colored area in both images represents the projected landing area given the current position and heading of the aircraft.

Figure 4 represents a similar situation to that in Figure 3 except with a large UAV. The probability model was adjusted to account for the large UAV for the different size and operating parameters of a larger UAV. Notice how the not-safe zones (red) increased in size due to the potential for impact reach.

\section{Integration of Probability Maps with Sensor DATA}

By treating the probability map as another sensor, we can use it in sensor fusion on the autonomous systems. By combining the information we receive from the GIS data (e.g., "The XYZ building is in front of me") with that of the other sensors (e.g., "Radar says there's something in front of me; the camera shows a window"), we can build an enhanced picture of the world. Because the extent of the GIS data is much greater than any of the robot's direct sensors, we can make 

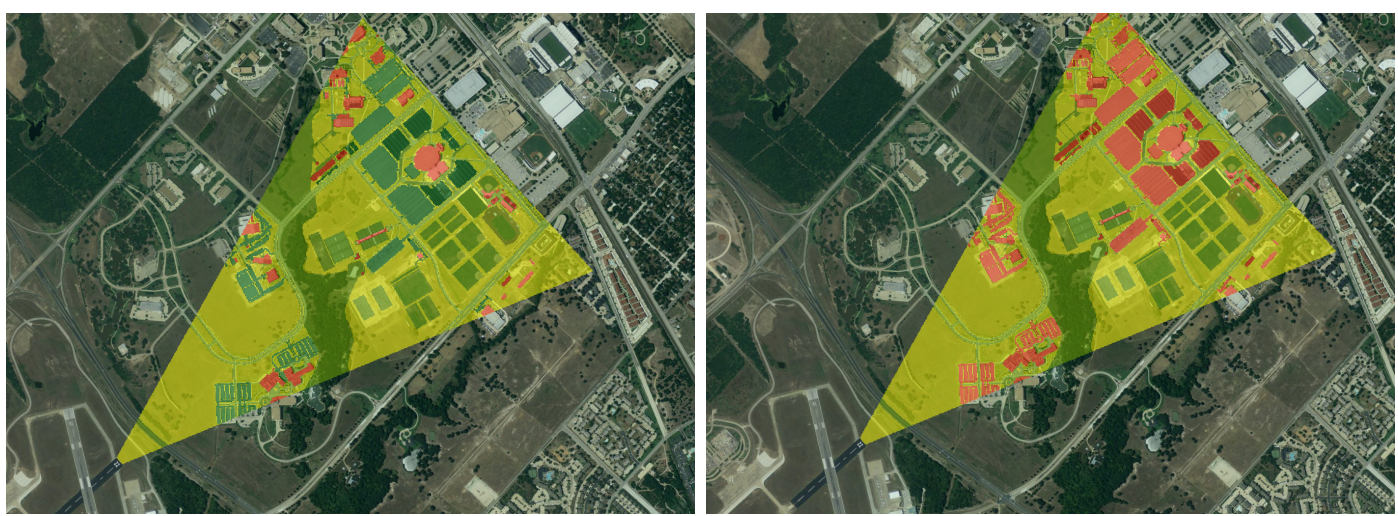

Fig. 3. Minimized impact probability maps for an emergency UAV landing at TAMU based on current location and possible trajectories: (a) small UAV during the weekday, (b) small UAV during the weekend.
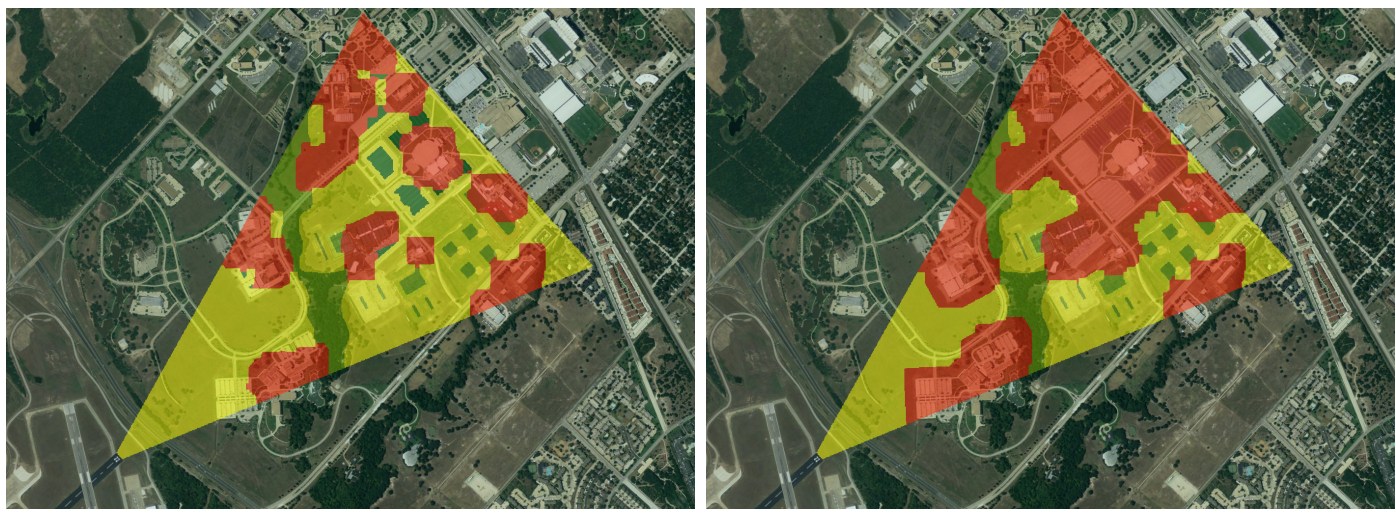

Fig. 4. Minimized impact probability maps for an emergency landing of a large UAV: (a) weekday, (b) weekend.

plans that are not limited to the current line of sight of the robot, but instead extend further into the world.

Additionally, once we have a probability map of our area of interest, we can use this information to facilitate robot navigation. By feeding the robot's position into the probability map, we can calculate a least-cost surface from that starting point. This use of the data allows the robot to determine leastcost paths from its current position to any other point on the map. By providing this capability on ArcGIS Server, the paths can be calculated dynamically and updated as the robot moves across the world, allowing us to offload some of the navigation tasks from the robot to the GIS service. Figure 5 presents the two images representing cost-surface maps for UGV navigation at TAMU. The image on the left is the leastcost path. The image on the right shows the adjusted path after a hazard has been detected. The yellow areas permit navigation according to the GIS information; the coloring fades to blue representing non-traversal. The robot's current position is marked by the red circle, along with the planned path (red line).

\section{DISCUSSION}

The architecture, incorporating ArcGIS components for geoprocessing, proved useful in constructing the minimal impact and cost-surface probability maps. Informal feedback from professionals in the military UAV and UGV fields confirmed the potential benefit of this information being shared with operators and mission planners. Additionally, it is relatively straightforward to modify weights and parameters to represent differently sized UAV platforms.

It is important to note that these maps should be considered just another type of sensor and one that is out of date. Because of the time required for collection of GIS data, the layers used do not reflect current conditions, but rather the state of the world as it was during the last survey. However, related efforts have demonstrated how robot system information may be used to update GIS data. For this effort, we make use of the GIS data available without updating; future work would involve GIS updating.

In addition, data obtained from different sources will likely have different ages. Generally, we would weight more recent information as being more valuable, but also need to consider the resolution of the data: perhaps it makes more sense to use a higher resolution data set that may be older.

While for the purposes of this effort, the composition function used was a simple weighted sum, it is by no means the only function that could be used. Because of the strong geoprocessing tools available, any function could be applied to the available map layers to produce results best suited to the particular scenario. 

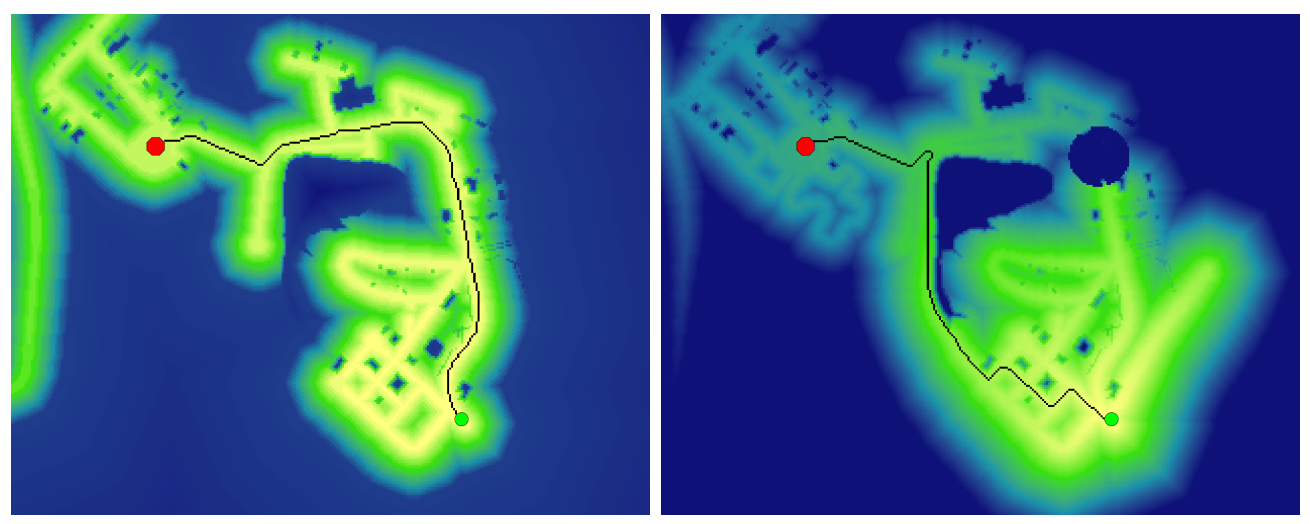

Fig. 5. Cost-surface maps for UGV navigation at TAMU based on the current location and intended destination: (a) least-cost path for the UGV, (b) least-cost path adjusted when hazard is marked.

\section{CONCLUSions AND Future Work}

This effort provided a proof of concept in how GIS data is useful for UGV navigation and UAV landing through producing cost surface and minimal impact probability maps. Informal feedback supported the benefit this information would bring to the users of UGV and UAV systems. Lessons learned from this effort, discussed in Section VI, dictate the future work.

Follow-on efforts will need to address: a) core architecture, b) user requirements, and c) interface for data interaction. We should consider the architecture design when evolving the ability to overlay and fuse GIS data for computing probabilistic maps. The design currently employs ArcGIS. Alternative designs may provide a more seamless means of building the probability maps. It is important to also consider integration within existing command and control environments.

The target users of this effort include the robot operators as well as mission planners. Mission planners are responsible for plotting resource utilization to meet mission objectives. A mission planner would need to identify an area of interest, requirements for the mission, and desirable or undesirable landing zones. These types of contextual information should be incorporated in the probability map construction.

Presenting the integrated GIS data to a user is also crucial. Another avenue for future work includes the design of a usercentered interface for command and control incorporating the map probability views using alternative interactions, such as multi-touch displays [14]. The richness of the map probability views may demand multi-touch to enhance data presentation.

\section{ACKNOWLEDGMENT}

The authors would like to thank the organizations who made their GIS data available and Dr. Richard Weatherly for his support. This effort was funded by The MITRE Corporation through the MITRE Innovation Program.

\section{REFERENCES}

[1] J. Peng, M. El Najjar, C. Cappelle, D. Pomorski, F. Charpillet, and A. Deeb, "A novel geo-localisation method using GPS, 3D-GIS and laser scanner for intelligent vehicle navigation in urban areas," in Advanced
Robotics, 2009. ICAR 2009. International Conference on. IEEE, 2009, pp. 1-6.

[2] H. Mori and S. Kotani, "Robotic travel aid for the blind: HARUNOBU6," in European Conference on Disability, Virtual Reality, and Assistive Technology, 1998.

[3] S. Shair, J. Chandler, V. Gonzalez-Villela, R. Parkin, and M. Jackson, "The use of aerial images and gps for mobile robot waypoint navigation," Mechatronics, IEEE/ASME Transactions on, vol. 13, no. 6, pp. 692-699, 2008.

[4] C. Brenner and B. Elias, "Extracting landmarks for car navigation systems using existing GIS databases and laser scanning," International archives of photogrammetry remote sensing and spatial information sciences, vol. 34, no. 3/W8, pp. 131-138, 2003.

[5] P. Bonnifait, M. Jabbour, and V. Cherfaoui, "Autonomous navigation in urban areas using GIS-managed information," International Journal of Vehicle Autonomous Systems, vol. 6, no. 1, pp. 83-103, 2008.

[6] C. Cappelle, M. Najjar, D. Pomorski, and F. Charpillet, "Intelligent Geolocalization in Urban Areas Using Global Positioning Systems, ThreeDimensional Geographic Information Systems, and Vision,” Journal of Intelligent Transportation Systems, vol. 14, no. 1, pp. 3-12, 2010.

[7] L. Bai and Y. Wang, "Fusing image, GPS and GIS for road tracking using multiple condensation particle filters," in Intelligent Vehicles Symposium, 2008 IEEE. IEEE, 2008, pp. 162-167.

[8] S. Renault, A. Le Meur, and D. Meizel, "GPS/GIS localization for management of vision referenced navigation in urban environments," in Intelligent Transportation Systems, 2005. Proceedings. 2005 IEEE. IEEE, 2005, pp. 608-613.

[9] J. Mirats Tur, C. Zinggerling, and A. Corominas Murtra, "Geographical information systems for map based navigation in urban environments," Robotics and Autonomous Systems, vol. 57, no. 9, pp. 922-930, 2009.

[10] M. Kais, S. Dauvillier, A. De La Fortelle, I. Masaki, and C. Laugier, "Towards Outdoor Localization Using GIS, Vision System andStochastic Error Propagation," 2004.

[11] J. Meguro, K. Ishikawa, T. Hashizume, J. Takiguchi, I. Noda, and M. Hatayama, "Disaster information integration into geographic information system using rescue robots," in Proc IEEE/RSJ Int Conf on Intelligent Robots and Systems (IROS2006), 2006.

[12] A. Sanfeliu and J. Andrade-Cetto, "Ubiquitous networking robotics in urban settings," in Proceedings of the IEEE/RSJ IROS Workshop on Network Robot Systems, 2006, pp. 14-18.

[13] M. Jun and R. D'Andrea, "Probability map building of uncertain dynamic environments with indistinguishable obstacles," in American Control Conference, 2003. Proceedings of the 2003, vol. 4. IEEE, 2003, pp. 3417-3422.

[14] M. Micire, "Multi-touch interaction for robot command and control," Ph.D. dissertation, University of Massachusetts Lowell, 2010.

[15] H. Yanco, M. Micire, J. Drury, and J. Casper, "Improving command and control: how new interaction methods will help," University of Massachusetts Lowell, Tech. Rep., 2008. 